\title{
COMPLEXIFICATION OF REAL ANALYTIC GROUPS
}

\author{
BY \\ G. HOCHSCHILD( ${ }^{(1)}$
}

In dealing with the structure and representation theory of a given real analytic group $G$, one is led to ask whether or not there exists a continuous injective, or at least locally injective, homomorphism of $G$ into a complex analytic group. The most convenient tool for attacking this question is the universal complexification of $G$. This is a continuous homomorphism $\gamma$ of $G$ into a complex analytic group $G^{+}$with the property that, for every continuous homomorphism $\eta$ of $G$ into a complex analytic group $H$, there is one and only one complex analytic homomorphism $\eta^{+}$of $G^{+}$into $H$ such that $\eta^{+} \circ \gamma=\eta$. Clearly, any two universal complexifications of $G$ are equivalent in the evident sense.

The standard construction of $\gamma: G \rightarrow G^{+}$is as follows. For any real or complex Lie algebra $L$, denote by $\mathscr{S}(L)$ the simply connected real or complex analytic group whose Lie algebra is $L$. If $\mathscr{L}(G)$ denotes the Lie algebra of $G$ then $\mathscr{S}(\mathscr{L}(G))$ is the universal covering group of $G$, and we denote the kernel of the covering epimorphism $\mathscr{S}(\mathscr{L}(G)) \rightarrow G$ by $F$, i.e., $F$ is the fundamental group of $G$. Let $R$ and $C$ stand for the fields of the real and the complex numbers, respectively, and let $\sigma$ denote the continuous homomorphism of $\mathscr{S}(\mathscr{L}(G))$ into $\mathscr{S}\left(\mathscr{L}(G) \otimes_{R} C\right)$ whose differential is the canonical injection of $\mathscr{L}(G)$ into its complexification $\mathscr{L}(G) \otimes_{R} C$. Considering the adjoint representation of $\mathscr{S}\left(\mathscr{L}(G) \otimes_{R} C\right)$, one sees immediately that $\sigma(F)$ lies in the center of $\mathscr{S}\left(\mathscr{L}(G) \otimes_{R} C\right)$, whence the same is true for the smallest closed complex Lie subgroup, $F^{*}$ say, of $\mathscr{S}\left(\mathscr{L}(G) \otimes_{R} C\right)$ that contains $\sigma(F)$. We define $G^{+}$as the complex analytic group $\mathscr{S}\left(\mathscr{L}(G) \otimes_{R} C\right) / F^{*}$ and $\gamma$ as the continuous homomorphism $G \rightarrow G^{+}$that is obtained from $\sigma$ by passing to the factor groups $\bmod F$ and $\bmod F^{*}$. It is easy to verify that this is actually a universal complexification of $G$.

Our original question now becomes the question of whether or not $\gamma$ is injective or locally injective, i.e., of whether or not the kernel of $\gamma$ is trivial or discrete. Let us note first that $\sigma(\mathscr{S}(\mathscr{L}(G)))$ is always a closed real analytic subgroup of $\mathscr{S}\left(\mathscr{L}(G) \otimes_{R} C\right)$. Indeed, the complex conjugation of $\mathscr{L}(G) \otimes_{R} C$ induced from that of $C$ is the differential of a real analytic involution $\alpha$ of $\mathscr{S}\left(\mathscr{L}(G) \otimes_{R} C\right)$, and $\sigma(\mathscr{S}(\mathscr{L}(G)))$ is evidently the connected component of the identity in the $\alpha$-fixed subgroup. It follows that $F^{*}=\sigma(F)$ whenever $\sigma(F)$ is discrete in $\sigma(\mathscr{S}(\mathscr{L}(G)))$; for then $\sigma(F)$ is a discrete central subgroup of $\mathscr{S}\left(\mathscr{L}(G) \otimes_{R} C\right)$.

Received by the editors February 25, 1966.

${ }^{(1)}$ Thanks are due to Nagayoshi Iwahori whose comments and suggestions have led to considerable improvements in the presentation of the results of this paper. 
The situation is very simple in the case where $G$ is solvable. In that case, we may view $\mathscr{S}\left(\mathscr{L}(G) \otimes_{R} C\right)$ as a solvable simply connected real analytic group. It is a standard result (due to $\mathrm{C}$. Chevalley) that every analytic subgroup of such a group is closed and simply connected; cf. [1, Chapter XII, Theorem 2.2]. Hence, in the present case, $\sigma$ is a topological group isomorphism of $\mathscr{S}(\mathscr{L}(G)$ ) onto its closed image in $\mathscr{S}\left(\mathscr{L}(G) \otimes_{R} C\right)$. By our above remark, we have therefore $F^{*}=\sigma(F)$, whence $\gamma$ is injective. Thus $\gamma$ is injective whenever $G$ is solvable.

It is almost evident that $\gamma$ is injective (locally injective) whenever $G$ has a faithful (locally faithful) finite-dimensional continuous representation; cf. [1, Chapter XVII, §5] (on line 20, p. 205, loc. cit., read "group covering, whose kernel lies in $F$," for "topological group isomorphism"). The result for the solvable case shows that the converse of this is false; for it is well known that there are solvable real analytic groups having no locally faithful finite-dimensional continuous representations.

By contrast, if $G$ is semisimple then the kernel of $\gamma$ coincides with the universal representation kernel of $G$, i.e., every finite-dimensional continuous representation of $G$ is trivial on the kernel of $\gamma$, and there exists such a representation of $G$ whose kernel is precisely the kernel of $\gamma$ [1, Chapter XVII, Theorem 3.3]. Since a semisimple real analytic group always has a locally faithful finite-dimensional continuous representation (the adjoint representation), it is therefore clear from the above that if $\gamma: G \rightarrow G^{+}$is not locally injective then $G$ can be neither solvable nor semisimple. The simplest example of such a group is given later on. Observe that, because of the existence of the complexified adjoint representation, the kernel of $\gamma$ is always contained in the center of $G$.

We shall require two essentially well-known lemmas, which we include here for the convenience of the reader.

LEMMA 1. Let $G$ be a real analytic group, and let $S$ be a semisimple analytic subgroup of $G$. If the center of $S$ is finite then $S$ is closed in $G$.

Proof. Replacing $G$ with the closure of $S$ in $G$, we reduce this lemma to the case where $S$ is dense in $G$, which we shall now assume. Consider the adjoint representation of $G$. Since $\mathscr{L}(S)$ is stable under the adjoint action of $S$ and since $S$ is dense in $G$, it follows that $\mathscr{L}(S)$ is $G$-stable, whence $\mathscr{L}(S)$ is an ideal of $\mathscr{L}(G)$. Since $\mathscr{L}(S)$ is semisimple, this implies that $\mathscr{L}(G)$ is the direct sum of $\mathscr{L}(S)$ and a complementary ideal, which we write as $\mathscr{L}(A)$, where $A$ is the corresponding analytic subgroup of $G$. Since $\mathscr{L}(S)$ annihilates $\mathscr{L}(A)$, the elements of $\mathscr{L}(A)$ are left fixed by $S$ under the adjoint representation and hence are left fixed also by $G$. Hence $A$ lies in the center of $G$ (and, in fact, is the connected component of the identity in the center of $G$ ). Now consider the multiplication map of the direct product of the analytic groups $S$ and $A$ into the analytic group $G$. This is clearly a surjective analytic homomorphism, and therefore an open map. Moreover, the kernel of our homomorphism is finite, because $A \cap S$ lies in the finite center of $S$. 
This implies that our homomorphism is also a closed map, whence it is clear that $S$ is closed in $G$.

Lemma 2. Let $G$ be a real analytic group, $A$ an analytic subgroup of $G$. Let $N$ be a closed subgroup of $G$ that is normalized by $A$. Suppose that $A N$ and $A \cap N$ are closed in $G$. Then $A$ is closed in $G$.

Proof. Consider the semidirect product $(A /(A \cap N)) \cdot(N /(A \cap N))$ formed from the analytic subgroup $A /(A \cap N)$ and the closed normal subgroup $N /(A \cap N)$ of the Lie group $(A N) /(A \cap N)$ by using the conjugation action of $A$ on $N$. The multiplication map is a continuous and bijective homomorphism of this semidirect product onto $(A N) /(A \cap N)$. As a closed subgroup of the analytic group $G$, the group $N$ is the union of a countable family of compact subsets. Hence the same is true for the factor group $N /(A \cap N)$, and hence also for our semidirect product. By a well-known theorem of Pontrjagin's, it follows that the above continuous and bijective homomorphism is actually an isomorphism of topological groups. Hence it is clear that $A /(A \cap N)$ is closed in $(A N) /(A \cap N)$. This implies that $A$ is closed in $A N$ and therefore also in $G$, so that Lemma 2 is proved.

THEOREM 3. Let $G$ be a real analytic group, and let $\gamma: G \rightarrow G^{+}$be the universal complexification of $G$. Then $\mathscr{L}(\gamma(G))$ is a real form of $\mathscr{L}\left(G^{+}\right)$, and $G^{+}$has a real analytic involution $\tau$, whose differential is the corresponding complex conjugation of $\mathscr{L}\left(G^{+}\right)$, such that $\gamma(G)$ is the connected component of the identity in the $\tau$-fixed subgroup. Moreover, the homogeneous space $G^{+} / \gamma(G)$ is simply connected.

THEOREM 4. In the notation of Theorem 3, and with $D$ denoting the kernel of $\gamma$, and $S$ a maximal semisimple analytic subgroup of $G$, and $P$ the radical of $G$, the following five conditions are equivalent:

(1) $\gamma$ is locally injective;

(2) $S$ is closed in $G$;

(3) $S \cap D$ is closed in $G$;

(4) the restriction of $\gamma$ to $P$ is locally injective;

(5) $D$ coincides with the kernel of the universal complexification of $S$.

If $\gamma$ is locally injective then the canonical map $S^{+} \rightarrow G^{+}$is a complex analytic isomorphism of $S^{+}$onto a closed complex analytic subgroup of $\mathrm{G}^{+}$. If the restriction of $\gamma$ to $P$ is injective then the canonical map $P^{+} \rightarrow G^{+}$is a complex analytic isomorphism of $P^{+}$onto the radical of $G^{+}$, so that $G^{+}=S^{+} P^{+}$.

Proof. It is clear from the defining properties of a universal complexification that we may identify $(G / D)^{+}$with $G^{+}$and that then the canonical map $G / D \rightarrow$ $(G / D)^{+}$is the map induced from $\gamma$ in the natural way. Hence it suffices to prove Theorem 3 in the case where $\gamma$ is injective. The proof of this case will be absorbed in the proof of Theorem 4.

Since the center of a semisimple complex analytic group is finite, it is clear that the center of $\gamma(S)$ is finite. From Lemma 1, we have, therefore, that $\gamma(S)$ is closed 
in $G^{+}$, and hence that $S D$ is closed in $G$. Now suppose that $S \cap D$ is closed in $G$. Then we may apply Lemma 2 to conclude that $S$ is closed in $G$. Thus condition (3) of Theorem 4 implies condition (2), so that (3) and (2) are equivalent. It is evident that (1) implies (3) and (4).

Next, we show that (4) implies (1). Consider the canonical epimorphism $G \rightarrow G / D$. The image of $P$ is evidently the radical of $G / D$, and (4) implies that the radical of $G / D$ has the same dimension as the radical $P$ of $G$. The image of $S$ in $G / D$ is clearly a maximal semisimple analytic subgroup of $G / D$. Since $D$ lies in the center of $G$, the group $S \cap D$ lies in the center of $S$ and is therefore a discrete subgroup of the analytic group $S$. Hence the image of $S$ in $G / D$ is an analytic subgroup of the same dimension as $S$. Since the dimension of an analytic group is the sum of the dimension of any maximal semisimple analytic subgroup and that of the radical, it follows that $G / D$ is of the same dimension as $G$, which shows that $D$ is discrete in $G$. Thus (4) implies (1).

Since (5) evidently implies (3) and (1), the equivalence of the five conditions of Theorem 4 will be established as soon as we have shown that (2) implies (5). Let $\pi: P^{\circ} \rightarrow P$ be the universal covering of $P$. The adjoint action of $S$ on the Lie algebra of $P$ determines an action of $S$ on $P^{\circ}$ in the canonical fashion. We form the corresponding semidirect product $S \cdot P^{\circ}$, and we let $Q$ stand for the kernel of the epimorphism $S \cdot P^{\circ} \rightarrow S P=G$ that is induced from $\pi$ and the multiplication of $G$. Evidently, $Q$ consists of the elements $\left(\pi(t), t^{-1}\right)$ with $t$ in $\pi^{-1}(S \cap P)$. The action of $S$ on $\mathscr{L}(P)$ extends canonically to a representation of $S$ by automorphisms of the complex Lie algebra $\mathscr{L}(P) \otimes_{R} C$. Clearly, $\left(P^{\circ}\right)^{+}=\mathscr{S}\left(\mathscr{L}(P) \otimes_{R} C\right)$. Our representation of $S$ on $\mathscr{L}(P) \otimes_{R} C$ defines a complex analytic representation of $S^{+}$by automorphisms of this Lie algebra, corresponds to a unique representation of $S^{+}$by complex analytic group automorphisms of $\left(P^{\circ}\right)^{+}$. If $S^{+} \cdot\left(P^{\circ}\right)^{+}$ is the corresponding semidirect product then the canonical maps $S \rightarrow S^{+}$and $P^{\circ} \rightarrow\left(P^{\circ}\right)^{+}$combine to yield a continuous homomorphism $\delta: S \cdot P^{\circ} \rightarrow S^{+} \cdot\left(P^{\circ}\right)^{+}$. Since the map $P^{\circ} \rightarrow\left(P^{\circ}\right)^{+}$is injective, the kernel of $\delta$ coincides with the kernel, $K$ say, of the canonical homomorphism $S \rightarrow S^{+}$.

Since $S \cap P$ is contained in the center $Z(S)$ of $S$, we have $Q \subset Z(S) \cdot \pi^{-1}(S \cap P)$. The image of $Z(S)$ in $S^{+}$lies in the finite center $Z\left(S^{+}\right)$of the semisimple complex analytic group $S^{+}$. Hence $\delta(Q) \subset Z\left(S^{+}\right) \cdot \rho\left(\pi^{-1}(S \cap P)\right)$, where $\rho$ is the canonical homomorphism $P^{\circ} \rightarrow\left(P^{\circ}\right)^{+}$. Since $P^{\circ}$ is solvable, $\rho$ is a topological group isomorphism of $P^{\circ}$ onto a closed real analytic subgroup of $\left(P^{\circ}\right)^{+}$. Now suppose that $S$ is closed in $G$. Then $S \cap P$ is a closed and hence discrete subgroup of $P$. Since $\pi$ is a covering, it follows that $\pi^{-1}(S \cap P)$ is discrete in $P^{\circ}$. Hence $\rho\left(\pi^{-1}(S \cap P)\right)$ is a discrete subgroup of the closed subgroup $\rho\left(P^{\circ}\right)$ of $\left(P^{\circ}\right)^{+}$, so that $\rho\left(\pi^{-1}(S \cap P)\right)$ is discrete also in $\left(P^{\circ}\right)^{+}$. Since $Z\left(S^{+}\right)$is finite, it follows that $Z\left(S^{+}\right) \cdot \rho\left(\pi^{-1}(S \cap P)\right)$ is discrete in $S^{+} \cdot\left(P^{\circ}\right)^{+}$, whence also the subgroup $\delta(Q)$ is discrete in $S^{+} \cdot\left(P^{\circ}\right)^{+}$.

Since $Z(S)$ is discrete in $S$ and $\pi^{-1}(S \cap P)$ is discrete in $P^{\circ}$, it is clear that $Z(S) \cdot \pi^{-1}(S \cap P)$ is discrete in $S \cdot P^{\circ}$. Hence also the subgroup $Q$ is discrete in 
$S \cdot P^{\circ}$. Since $Q$ is normal in $S \cdot P^{\circ}$, it is therefore central in $S \cdot P^{\circ}$. It follows that $\delta(Q)$ centralizes $\delta\left(S \cdot P^{\circ}\right)$. But the Lie algebra of $\delta\left(S \cdot P^{\circ}\right)$ spans the Lie algebra of $S^{+} \cdot\left(P^{\circ}\right)^{+}$over $C$. Hence it is clear that $\delta(Q)$ lies in the center of $S^{+} \cdot\left(P^{\circ}\right)^{+}$.

Now $\left(S^{+} \cdot\left(P^{\circ}\right)^{+}\right) / \delta(Q)$ is a complex analytic group, and the composite of $\delta$ with the canonical epimorphism $S^{+} \cdot\left(P^{\circ}\right)^{+} \rightarrow\left(S^{+} \cdot\left(P^{\circ}\right)^{+}\right) / \delta(Q)$ gives rise to a continuous homomorphism $\eta: G \rightarrow\left(S^{+} \cdot\left(P^{\circ}\right)^{+}\right) / \delta(Q)$. Since the complete inverse image of $\delta(Q)$ in $S \cdot P^{\circ}$ is $K Q$, the kernel of $\eta$ is precisely $K$. Since $\eta=\eta^{+} \circ \gamma$, this shows that the kernel of $\gamma$ is contained in $K$. On the other hand, it is evident that $K$ must be contained in the kernel of $\gamma$. Thus the kernel of $\gamma$ coincides with $K$, and we have shown that (2) implies (5). This completes the proof of the equivalence of the five conditions of Theorem 4.

For what remains to be proved, we assume that these conditions are satisfied, and we use the results obtained so far. It is clear from the above construction that $\eta$ satisfies the requirements for a universal complexification of $G$, so that we may conclude that $\eta^{+}$is an isomorphism of the complex analytic group $G^{+}$onto the complex analytic group $\left(S^{+} \cdot\left(P^{\circ}\right)^{+}\right) / \delta(Q)$. Since $\delta(Q)$ is discrete, this shows also that $\mathscr{L}(G)$, when identified with its image in $\mathscr{L}\left(G^{+}\right)$, is a real form of $\mathscr{L}\left(G^{+}\right)$.

We have $S^{+} \cap \delta(Q)=\delta(S \cap(K Q))=\delta(K)=(1)$. Transferring this information to $G^{+}$by means of the isomorphism $\eta^{+}$, we see that the canonical map $S^{+} \rightarrow G^{+}$ is injective. The image of $S^{+}$in $G^{+}$is a semisimple complex analytic subgroup of $G^{+}$. Hence its center is finite, and we may apply Lemma 1 to conclude that the image of $S^{+}$is closed in $G^{+}$. Thus the canonical map $S^{+} \rightarrow G^{+}$is a complex analytic isomorphism of $S^{+}$onto a closed complex analytic subgroup of $G^{+}$.

We know that $S^{+}$is the factor group of $\left(S^{\circ}\right)^{+}$modulo a finite central subgroup which lies in the image of $S^{\circ}$. Hence the real analytic involution of $\left(S^{\circ}\right)^{+}$whose differential is the complex conjugation of $\mathscr{L}(S) \otimes_{R} C$ induces a real analytic involution of $S^{+}$such that the image of $S$ in $S^{+}$is the connected component of the identity in the fixed subgroup. This real analytic involution of $S^{+}$evidently extends to a real analytic involution of $S^{+} \cdot\left(P^{\circ}\right)^{+}$whose differential is the natural complex conjugation of its Lie algebra $\mathscr{L}\left(S \cdot P^{\circ}\right) \bigotimes_{R} C$. The connected component of the identity in the corresponding fixed subgroup is clearly $\delta\left(S \cdot P^{\circ}\right)$. Hence we obtain a real analytic involution of $\left(S^{+} \cdot\left(P^{\circ}\right)^{+}\right) / \delta(Q)$ such that the connected component of the identity in the corresponding fixed subgroup is $\eta(G)$. Transporting this to $G^{+}$by means of the isomorphism $\eta^{+}$, we obtain a real analytic involution $\tau$ of $G^{+}$with the properties described in Theorem 3 .

Now it is clear that $\gamma(G)$ is closed in $G^{+}$, and we show that $G^{+} / \gamma(G)$ is simply. connected. We may identify $G^{+}$with $\gamma(G)^{+}$and, replacing $G$ with $\gamma(G)$, we may therefore assume that $\gamma$ is a topological group isomorphism of $G$ onto the closed subgroup $\gamma(G)$ of $G$. Now let us consider the commutative diagram

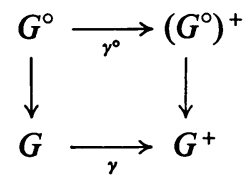


where the vertical map on the left is the universal covering, whose kernel is the fundamental group $F$ of $G$. Since $\gamma$ is injective, the kernel of $\gamma^{\circ}$ is contained in $F$. We know that $\gamma^{\circ}\left(G^{\circ}\right)$ is closed in $\left(G^{\circ}\right)^{+}$. Hence it is clear that $\gamma^{\circ}(F)$ is discrete in $\left(G^{\circ}\right)^{+}$, so that $G^{+}=\left(G^{\circ}\right)^{+} / \gamma^{\circ}(F)$. On the other hand, $\gamma(G)$ is the image of $\gamma^{\circ}\left(G^{\circ}\right)$ under the vertical map on the right, i.e., $\gamma(G)=\gamma^{\circ}\left(G^{\circ}\right) / \gamma^{\circ}(F)$. Thus we have $G^{+} / \gamma(G)=\left(G^{\circ}\right)^{+} / \gamma^{\circ}\left(G^{\circ}\right)$. Since $\left(G^{\circ}\right)^{+}$is simply connected and $\gamma^{\circ}\left(G^{\circ}\right)$ is connected, it follows from a standard topological result that $G^{+} / \gamma(G)$ is simply connected (cf. [1, Chapter IV, Exercise 1]), so that Theorem 3 is now established.

All that remains to be proved is the last statement of Theorem 4. Let $x$ be an element of $\left(P^{\circ}\right)^{+} \cap \delta(Q)$, and choose an element $y$ in $Q$ such that $x=\delta(y)$. Write $y=\left(\pi(t), t^{-1}\right)$ with $t$ in $\pi^{-1}(S \cap P)$. Since $x$ belongs to $\left(P^{\circ}\right)^{+}$, we must have $\delta(\pi(t))=1$, i.e., $\pi(t) \in K$. Now if $\gamma$ is injective on $P$ then $K \cap P$ is trivial, so that $\pi(t)=1$, i.e., $t$ lies in the fundamental group of $P$. Thus, identifying $P^{\circ}$ with its image in $\left(P^{\circ}\right)^{+}$, we have that $\left(P^{\circ}\right)^{+} \cap \delta(Q)$ is precisely the fundamental group of $P$. But the factor group of $\left(P^{\circ}\right)^{+}$modulo the fundamental group of $P$ is $P^{+}$. Hence it follows that the image of $\left(P^{\circ}\right)^{+}$in $\left(S^{+} \cdot\left(P^{\circ}\right)^{+}\right) / \delta(Q)$ is isomorphic, as a complex analytic group, with $P^{+}$. Transporting this to $G^{+}$by means of the isomorphism $\eta^{+}$, we conclude that the canonical image of $P^{+}$in $G^{+}$, which is evidently the radical of $G^{+}$, may be identified with $P^{+}$. This completes the proof of Theorems 3 and 4 .

The following is the simplest example of a real analytic group $G$ such that $\gamma: G \rightarrow G^{+}$is not locally injective. Let $L$ denote the Lie algebra consisting of the linear endomorphisms of trace 0 of $R^{2}$. Then $\mathscr{S}(L)$ is the universal covering group of the special real linear group $S L(2, R)$, and $\mathscr{S}\left(L \otimes_{R} C\right)$ is the special complex linear group $S L(2, C)$. The fundamental group, $A$ say, of $S L(2, R)$ is an infinite cyclic group, and it is precisely the kernel of the canonical homomorphism $\mathscr{S}(L) \rightarrow \mathscr{S}\left(L \otimes_{R} C\right)$. Let $\alpha: A \rightarrow Z$ be an isomorphism of $A$ onto the additive group $Z$ of the integers. For a real number $s$, let $s^{*}$ denote its canonical image in the 1-dimensional toroidal group $R / Z$. Let $B$ denote the discrete central subgroup of the direct product $\mathscr{S}(L) \times(R / Z)$ consisting of the elements $\left(a,(\alpha(a) r)^{*}\right)$, where $a$ ranges over $A$ and where $r$ is a fixed irrational real number. Put $G=(\mathscr{S}(L) \times(R / Z)) / B$. From the fact that the projection image of $B$ in $R / Z$ is dense in $R / Z$ one sees easily that the kernel of $\gamma$ is precisely the image of $A \times(R / Z)$ in $G$, and that $G^{+}=S L(2, C)$.

For the purposes of representation theory, it is of interest to determine when the universal complexification $G^{+}$of a real analytic group $G$ has a faithful finitedimensional complex analytic representation. A criterion for this is given by the following result.

THEOREM 5. Let $\gamma: G \rightarrow G^{+}$be the universal complexification of a real analytic group $G$. Then $G^{+}$has a faithful finite-dimensional complex analytic representation whenever $G$ has a locally faithful finite-dimensional continuous representation.

Proof. Let $\rho$ be a locally faithful continuous representation of $G$ on a finitedimensional real vector space $V$. The existence of $\rho$ implies that $\gamma$ is locally injective 
so that, by Theorem 3 , we may identify $\mathscr{L}(G)$ with a real form of $\mathscr{L}\left(G^{+}\right)$, i.e., we may write $\mathscr{L}\left(G^{+}\right)=\mathscr{L}(G) \bigotimes_{R} C$. Let $\rho_{1}$ denote the canonical extension of $\rho$ to a representation of $G$ by complex linear automorphisms of $V \otimes_{R} C$. Then the differential of $\rho_{1}^{+}$is evidently the canonical extension of the differential of $\rho$ to a representation of $\mathscr{L}\left(G^{+}\right)$by linear endomorphisms of $V \otimes_{R} C$. It is clear from this that the differential of $\rho_{1}^{+}$is a faithful representation of $\mathscr{L}\left(G^{+}\right)$, so that $\rho_{1}^{+}$is a locally faithful complex analytic representation of $G^{+}$. Hence Theorem 5 will be established as soon as we have proved the following proposition.

Proposition 6. Let $H$ be a complex analytic group, and suppose that $H$ has a locally faithful finite-dimensional complex analytic representation. Then $H$ has a faithful finite-dimensional complex analytic representation.

Proof. The assumption means that there is a discrete central subgroup $D$ of $H$ such that $H / D$ has a faithful finite-dimensional complex analytic representation. Let us first deal with the case where $H / D$ is reductive, i.e., where, moreover, every finite-dimensional complex analytic representation of $H / D$ is semisimple. It is known that this is the case if and only if $H / D$ is the universal complexification of a compact real analytic group [1, Chapter XVII, Theorem 5.3]. Let $H^{\circ}$ denote the universal covering group of $H$. Our assumption implies that $H^{\circ}$ is a direct product $S \times V$, where $V$ is a complex vector group and $S$ is a semisimple complex analytic group. Since $H^{\circ}$ is also the universal covering group of $H / D$, there is a discrete central subgroup $E$ of $S \times V$ such that $H / D=(S \times V) / E$. Let $F$ be the projection image of $E$ in $S$. Then $F$ is finite, so that $F E$ is still a discrete central subgroup of $S \times V$. Moreover, $F E=F \times Y$, where $Y$ is the discrete subgroup $(F E) \cap V$ of $V$. Now $(S / F) \times(V / Y)$ is isomorphic with the factor group of $H / D$ modulo a finite central subgroup, whence it is easily seen that $(S / F) \times(V / Y)$ is reductive (use [1, Chapter XVII, Lemma 3.1]). Clearly, $V / Y$ is the connected component of the identity in the center of this reductive group, which implies that $V / Y$ is reductive [2, p. 91]. Hence $V / Y$ is a complex toroid. More precisely, $V$ may therefore be identified with $C^{n}$ in such a way that $Y$ becomes identified with the lattice of $C^{n}$ consisting of the points whose coordinates are integers.

Now $H$ is isomorphic with $(S \times V) / X$, where $X \subset E$. The canonical image of $V$ in $H$ is the connected component of the identity in the center of $H$. It is isomorphic with $V /(V \cap X)$. Since $V \cap X \subset Y$, it is clear from the above description of $V / Y$ that $V /(V \cap X)$, and thus the image of $V$ in $H$, is of the form $T \times U$, where $T$ is a complex toroid and $U$ is a complex vector group. Let $S^{*}$ denote the image of $S$ in $H$. Then $S^{*}$ is a semisimple complex analytic subgroup of $H$, and we have $H=S^{*}(T \times U)$. Moreover, $S^{*} \cap(T \times U)$ lies in the finite center of $S^{*}$, so that it must be contained in the maximum compact subgroup of $T \times U$, and hence must be contained in $T$. Hence $H$ is the direct product $\left(S^{*} T\right) \times U$. It is easily seen that $S^{*} T$ has a faithful finite-dimensional complex analytic representation, whence the same is true for $H$ (use [1, Chapter XVII, Lemma 3.1] in order to deal with $S^{*} T$ ). 
Now let us consider the general case. Since $H / D$ is a complex analytic linear group, it has a semidirect product decomposition $H / D=M \cdot K$, where $M$ is reductive and $K$ is a simply connected, solvable, complex analytic group, normal in $H / D$ [2, Theorem 4.2]. Let $K^{*}$ be the connected component of the identity in the complete inverse image of $K$ in $H$. Then, since $K$ is simply connected and $D$ is discrete, the canonical epimorphism $H \rightarrow H / D$ restricts to a complex analytic isomorphism of $K^{*}$ onto $K$. Now consider the factor group $H / K^{*}$. This contains $\left(D K^{*}\right) / K^{*}$ as a discrete central subgroup, and the corresponding factor group is isomorphic with $M$, and therefore reductive. From what we have already proved, we know therefore that $H / K^{*}$ has a faithful finite-dimensional complex analytic representation. Hence we have $H / K^{*}=L \cdot P$, where $L$ is reductive and $P$ is simply connected, solvable, and normal in $H / K^{*}$. Now $P$ may be written in the form $Q / K^{*}$, where $Q$ is the connected component of the identity in the inverse image of $P$ in $H$. Clearly, $Q$ is a solvable closed normal complex analytic subgroup of $H$. Moreover, since $Q / K^{*}$ and $K^{*}$ are simply connected, $Q$ is simply connected. Now $H / Q$ is isomorphic with $L$, and hence reductive. By [2, Theorem 3.6], we may therefore conclude that $H$ has a faithful finite-dimensional complex analytic representation. This establishes Proposition 6, and therefore also Theorem 5.

\section{REFERENCES}

1. G. Hochschild, The structure of Lie groups, Holden-Day, San Francisco, 1965.

2. G. Hochschild and G. D. Mostow, Representations and representative functions of Lie groups. III, Ann. of Math. 70 (1959), 85-100.

\section{UNIVERSITY OF CALIFORNIA,} Berkeley, California 